Eye (1990) 4, 138-144

\title{
The Use of Contact Lenses to Correct Aphakia in a Clinical Trial of Cataract Management
}

\author{
OXFORD CATARACT TREATMENT AND EVALUATION TEAM (OCTET) \\ The Eye Hospital, Oxford
}

\begin{abstract}
Summary
In a prospective study, 110 eligible patients (111 eyes) were randomly selected to have contact lenses for aphakia (CL). The lens of first choice was a daily-wear hard (DWH) PMMA contact lens. Extended-wear (XW) lenses were fitted to those who failed with DWH lenses. The numbers in the two groups were approximately equal. Eighty per cent of eyes were successfully wearing CLs six months post-operatively and $72 \%$ were still wearing them after five years.

The mean age of both groups of patients was greater than 70 years but the DWH group was significantly younger $(\mathbf{p}<\mathbf{0 . 0 0 1})$. However, $\mathrm{XW}$ lens wearers suffered more serious complications, needed more follow-up and more lens replacements. There were also more drop-outs from the XW group compared to the DWH group.
\end{abstract}

Before the acceptance of intraocular lens implantation as a routine procedure in cataract surgery, the use of contact lenses provided an important alternative in the correction of aphakia without the distortion of aphakic spectacles. Although most contraindications to lens implantation have become relative, there are still situations in which an intraocular lens may not be used, for example, in cases of trauma involving tissue loss or in young patients below an age limit set by the surgeon. It is also debatable whether one should implant an anterior chamber (AC) lens at the time of vitreous loss when attempting extracapsular extraction where, after vit- rectomy, the support for a posterior chamber lens may be lacking. A surgeon accustomed to using posterior chamber lenses and extracapsular surgery may not be familiar with AC lenses and to use an AC lens in a complicated situation is to increase the risks of further complications. Therefore, there are still strong indications for contact lenses and a knowledge of the long-term tolerance is important.

This paper reports the results of a clinical trial in which a cohort of patients willing to use contact lenses for the correction of aphakia has been followed for a minimum of five years.

Participants: Principal Investigators, H. Cheng, K. McPherson;* Contributing Surgeons, H. Cheng, D. Boase, G. Bell; Research Fellows, N. Price, P. Jacobs, M. Noble, S. MacLennan, A. K. Bates; Consultant in Contact Lenses, J. Kersley; † Computer Programmer, L. Jones; Research Assistants, C. Tagari, L. Wood; Photographer, D. Barbour; Contact Lens Practitioner, D. White; Optician (Optometrist), C. Lydon; Nurse Technician, A. Ambrose; Trial Administrator, I. Samuel.

Trial Location: The Eye Hospital, Oxford.

${ }^{*}$ Department of Community Medicine and General Practice, Oxford University, Oxford.

$\dagger 80$ Harley Street, London.

Correspondence to: Mr Hung Cheng FRCS, The Eye Hospital, Walton Street, Oxford OX2 6AN. 


\section{Patients and Method}

Patients comprised one 'arm' of a randomised controlled trial and the design of the clinical trial has been previously reported. ${ }^{1}$ The patients had simple 'senile' cataract and were free of other eye disease. They gave informed consent to having either a contact lens or a lens implant, and they also agreed to having the choice of treatment made by a randomisation procedure. One hundred and eleven eyes (of 110 patients) were randomised to have ICCE and CL. Intracapsular extraction was by standard ab externo technique and cryo extraction.

There were three cases of vitreous loss but no other serious intraoperative complication.

Routine follow-up visits were at two and four weeks post-operatively and CL fitting was attempted after six weeks.

The standard routine was to assess the patient for a hard contact lens at six to eight weeks to determine whether the eye had settled enough according to keratometry values and signs of inflammation. Teaching sessions were given to those who had reached a quiet and stable stage. The hard contact lens, where handling could easily be achieved was the lens of first choice. The others were fitted with extended-wear lenses.

The first choice daily-wear hard (DWH) lenses were of PMMA and the extended-wear (XW) lenses were of $71 \%$ water content 'Permalens'. In cases of intolerance, the protocol allowed the substitution of a daily-wear soft lens for a DWH lens and in the case of XW, a lens of a different make and water content (eg Sauflon 85). A small number of eyes which failed extended-wear were later fitted with daily-wear soft or gas permeable lens, the choice being made on empirical grounds.

\section{Fitting Technique}

For hard and extended-wear contact lenses followed standard principles. In hard fitting, PMMA material was used as the benefits of oxygen permeability in lenses of average centre thickness of $0.50 \mathrm{~mm}$ are negligible and the breakage rate is very much higher. The aim was to achieve an alignment fit and a C3 configuration was most commonly used, although a toric periphery was sometimes added to aid stability. A front optic reduction of $8.0 \mathrm{~mm}$ was standard and provided a measure of lid hitch-up to give good lens positioning.

The fitting of extended-wear lenses aimed for 1-2 mm of movement after the settling period and the average diameter was 14.0 $14.5 \mathrm{~mm}$. These patients were not taught handling techniques as they had already failed a 'teaching evaluation'.

\section{Follow-up schedule}

Hard Lenses - patients were reassessed one month after teaching then at six months and annually. Lenses were replaced for breakage or loss; or, if there were symptoms attributable to poor fit.

Extended-Wear-patients were seen the day after fitting and at regular intervals of two or three months until a routine was established. Lens cleaning took place at scheduled visits and lenses were replaced for loss or wear and tear.

A record was kept of all patient visits. Emergency visits were defined as those initiated by the patient because of some imperative need as distinct from routine visits required for examination and after care of the lens. Annually, each eye had a detailed examination and the visual acuity was obtained, with suitable spectacle over correction, by an optometrist who was not the lens fitter.

Complications were classed as 'trivial' if there was no threat to sight or to continuance of contact lens wear, but regarded as 'serious' if either sight threatening or if contact lens wear had to be discontinued for more than a few days. Serious complications included; corneal abrasion, corneal abscess, infection (non-abscess), progressive limbal vascularisation, marked decentration of lens, and persistent punctate epithelial erosions. Infection included symptomatic blepharitis, severe conjunctivitis and keratoconjunctivitis with or without ulceration.

With three exceptions, when the fellow eye developed a cataract which reduced the ability of the patient to manage the contact lens for the first eye, a lens implant procedure was carried out for the second eye.

\section{Results}

For the first year, all the patients, except two, 
Table I Number of patients wearing contact lenses of different styles

\begin{tabular}{lcccccc}
\hline & 6 months & 1 year & 2 years & 3 years & 4 years & 5 years \\
\hline Daily-wear (DW) hard + soft & $43+3$ & $36+6$ & $35+6$ & $31+6$ & $28+5$ & $26+4$ \\
Extended-wear (XW) & 46 & 51 & 43 & 37 & 29 & 27 \\
DW $\rightarrow$ XW in whole period & N/A & 3 & 3 & 1 & 1 & 0 \\
XW $\rightarrow$ *Daily-wear in whole period & 0 & 0 & $2(2)$ & $4(3)$ & $4(3)$ & $4(3)$ \\
$\%$ not wearing & 14 & 12 & 16 & 22 & 29 & 28 \\
$\%$ dead & 2 & 4 & 7 & 9 & 14 & 21 \\
\hline
\end{tabular}

* Gas permeable and Daily-wear soft. Numbers in brackets refer to gas permeable lenses.

$\mathrm{N} / \mathrm{A}=$ not applicable

were phakic or pseudophakic in the second eye. Also, up to the first year point, $57 \%$ of the patients had $6 / 12$ visual acuity or better in the second eye; $17 \%$ of patients required cataract surgery in the second eye and all, but two of these, had received a lens implant (one of the two was actually a failed implant). After five years, $64 \%$ of the fellow eyes had had cataract surgery.

At entry to the study the mean age of patients in the whole group was 72.5 years (range 55-87). The mean age of the dailywear patients was 70.8 years (range 58-81) which was significantly different $(\mathrm{p}<0.05)$ from that of the extended-wear patients whose mean was 73.7 years (range 56-85). After that point the difference in mean age tended to increase and after the one year point there was a highly significant difference in age between the two groups of patients $(\mathrm{p}<0.005)$ at each time point of assessment.

The visual acuity of the daily-wear patients was consistently better than the group with extended-wear lenses. Table II shows that the VA was better at one, three or five years whether the level for comparison chosen was $6 / 6$ or $6 / 12$.

The proportion of the eyes in both groups with $6 / 6$ vision dropped in later years compared to the first year, although this trend was less apparent in the extended-wear group as the numbers became smaller.

We were able to fit $40 \%$ of eyes with dailywear hard (DWH) lenses and $42 \%$ with extended-wear lenses. After fitting, $5 \%$ of the eyes could not tolerate DWH lenses and were fitted with daily-wear soft lenses (DWS) which were well tolerated once fitted (Table I). Because DWS lens wearers comprised such a small group compared to those with
DWH and XW lenses, they were excluded from the analyses.

The number of failures un-able to wear the contact lens increased with time, reaching nearly $30 \%$ by year four and there were more failures in the extended-wear group than the daily-wear group (Table I).

After the first year no eye fitted with a DWH lens had to change to an XW lens but four eyes initially fitted with an XW lens reverted to daily-wear lenses of another material.

Once fitted, both types of lenses were initially well tolerated and the mean number of weeks lost from wearing the lenses was small and was comparable in the two groups (Table III). Extended-wear lenses needed more attention (especially for lens cleaning) than the DWH lens group: the mean number of hospital visits made by each patient with XW was eleven in the first year though this dropped to approximately once evey two months for the five-year period. The mean

Table II The number of eyes with visual acuity at different levels

\begin{tabular}{cccc}
\hline $\begin{array}{c}\text { Time } \\
\text { in years }\end{array}$ & $\begin{array}{c}\text { Level } \\
\text { of VA }\end{array}$ & $\begin{array}{c}\text { Daily- } \\
\text { wear }\end{array}$ & $\begin{array}{c}\text { Extended- } \\
\text { wear }\end{array}$ \\
\hline \multirow{2}{*}{1} & $\geqslant 6 / 6$ & 28 & $7^{*}$ \\
& $6 / 9-6 / 12$ & 8 & 30 \\
& $<6-12$ & 0 & 4 \\
3 & $\geqslant 6 / 6$ & 22 & $5 \dagger$ \\
& $6 / 9-6 / 12$ & 5 & 24 \\
& $<6 / 12$ & 3 & 8 \\
5 & $\geqslant 6-6$ & 14 & $7 \ddagger$ \\
& $6 / 9-6 / 12$ & 11 & 13 \\
& $<6 / 12$ & 1 & 6 \\
\hline
\end{tabular}

\footnotetext{
Results of $x^{2}$ test:

${ }^{*} \mathrm{p}=0.0007$.

$+\mathrm{p}-4 \times 10^{-6}$

$\ddagger \mathrm{p}=<0.05$.
} 
Table III Tolerance of contact lenses: $D W H v s X W$

\begin{tabular}{lcccccc}
\hline & & 1 year & 2 years & 3 years & 4 years & 5 years \\
\hline Mean no of weeks lens not worn & DWH & 0 & 1 & 2 & 4 & 0 \\
between annual visits & XW & 1 & 4 & 1 & 3 & 6 \\
Mean no. of visits per patient & DWH & 8 & 9 & 11 & 12 & 13 \\
& XW & 11 & 17 & 22 & 28 & 32 \\
Emergency and patient-instigated & DWH & 6 & 6 & 7 & 7 & 7 \\
visits & XW & 7 & 9 & 10 & 12 & 12 \\
Mean no. of lens changes & DWH & 0.6 & 0.9 & 1.5 & 1.8 & 2.1 \\
& XW & 2 & 3 & 5 & 7 & 8 \\
\hline
\end{tabular}

DWH = Daily-wear hard.

$\mathrm{XW}=$ Extended-wear.

number of visits for DWH lenses was significantly less, especially for the later periods of the study. For those still wearing DWH lenses after the first year the mean number of visits per annum was between one and two.

Compared to the first year the number of unscheduled and emergency visits was considerably reduced in subsequent years but was significantly greater for the XW group.

The number of lenses replaced was up to four fold higher for the XW group and reached a mean of two lenses per person per year. However, some patients required more frequent changes such that for 13 patients, the total number of lenses required was 146 in five years (Table IV).

Serious complications were defined as events threatening sight or the continuance of contact lens wear. At each stage of assessment, XW lens wearers had more complications than the DWH group and the proportion also increased with time, leading to greater 'drop-outs' in lens wear (Table V). The commonest complications were infection and abrasion. Besides abscesses the ten cases of infection reported in the XW group in the five years comprised the following:

four blepharitis,

two conjunctivitis,

three kerato-conjunctivitis

(two with ulcers),

and one unspecified.

The only case of infection in the DWH group was one of kerato-conjunctivitis. The most serious complication was corneal abscess of which there were eight in five years, all of which occurred in the XW group (Table VI). Some of the complications occurred more than once and more than one complication occurred in some of the eyes (Table VI). If we excluded cases of punctate epithelial erosion from the list, the relative risk of serious complications is significantly higher for the XW group at each year of assessment.

\section{Discussion}

Contact lenses have been shown in previous

Table IV Number of eyes requiring lens changes

\begin{tabular}{|c|c|c|c|c|c|c|c|c|c|c|}
\hline & & \multicolumn{9}{|c|}{ Number of lenses required } \\
\hline & & 0 & 1 & 2 & 3 & 4 & 5 & 6 & 7 & $>8$ \\
\hline Year 1 & $\begin{array}{c}\text { DWH } \\
\text { XW }\end{array}$ & $\begin{array}{r}19 \\
6\end{array}$ & $\begin{array}{l}13 \\
13\end{array}$ & $\begin{array}{r}3 \\
16\end{array}$ & $\begin{array}{l}1 \\
6\end{array}$ & $\begin{array}{l}0 \\
5\end{array}$ & $\begin{array}{l}0 \\
4\end{array}$ & $\begin{array}{l}0 \\
1\end{array}$ & $\begin{array}{l}0 \\
0\end{array}$ & $\begin{array}{l}0 \\
0\end{array}$ \\
\hline Year 3 & $\begin{array}{c}\text { DWH } \\
\text { XW }\end{array}$ & $\begin{array}{l}7 \\
1\end{array}$ & $\begin{array}{r}16 \\
1\end{array}$ & $\begin{array}{l}3 \\
4\end{array}$ & $\begin{array}{l}1 \\
8\end{array}$ & $\begin{array}{l}3 \\
4\end{array}$ & $\begin{array}{l}0 \\
5\end{array}$ & $\begin{array}{l}0 \\
6\end{array}$ & $\begin{array}{l}0 \\
5\end{array}$ & $\begin{array}{l}1 \\
3\end{array}$ \\
\hline Year 5 & $\begin{array}{c}\text { DWH } \\
\text { XW }\end{array}$ & $\begin{array}{l}5 \\
0\end{array}$ & $\begin{array}{l}9 \\
0\end{array}$ & $\begin{array}{l}5 \\
0\end{array}$ & $\begin{array}{l}2 \\
0\end{array}$ & $\begin{array}{l}3 \\
3\end{array}$ & $\begin{array}{l}0 \\
5\end{array}$ & $\begin{array}{l}1 \\
2\end{array}$ & $\begin{array}{l}0 \\
4\end{array}$ & $\begin{array}{c}1 \\
13^{*}\end{array}$ \\
\hline
\end{tabular}

* These 13 eyes required a total of 146 new lenses.

DWH $=$ Daily-wear hard.

$\mathrm{XW}=$ Extended-wear. 
Table $\mathbf{V}$ Cumulative complications of DWH and XW lenses

\begin{tabular}{|c|c|c|c|c|c|c|}
\hline & \multicolumn{6}{|c|}{ Number of lenses post-op } \\
\hline & \multicolumn{2}{|c|}{1} & \multicolumn{2}{|c|}{3} & \multicolumn{2}{|c|}{5} \\
\hline & $D W H$ & $X W$ & $D H W$ & $X W$ & $D W H$ & $X W$ \\
\hline Cumulative no. of eyes with 'serious' complications & 3 & 7 & 7 & 19 & 8 & 23 \\
\hline No. of eyes wearing $\mathrm{CL}$ at timepoint & 36 & 51 & 31 & 37 & 26 & 27 \\
\hline No. of 'dropouts' at timepoint & 1 & 8 & 4 & 15 & 4 & 18 \\
\hline
\end{tabular}

DWH $=$ Daily-wear hard.

$\mathrm{XW}=$ Extended-wear.

studies to be adequate alternatives to IOLs ${ }^{2,3}$ as devices for aphakic correction. It has been suggested that hard lens wearers may have a large drop out rate ${ }^{4}$ but the early promise of extended-wear lenses ${ }^{5,6}$ has not been fulfilled. ${ }^{7}$ In the largest study with the longest follow-up to date, extended-wear lenses compare unfavourably especially in terms of serious complications. ${ }^{7,8}$

As well as being truly prospective, our study is unique in having the longest follow-up to date with a minimum period of five years for each patient and having only two cases lost to follow-up ( $2 \%)$. Furthermore, the patients were selected to have contact lenses by a randomisation procedure of cataract management.

Our findings confirm those of Graham et $a l .{ }^{8}$ that XW lenses caused significantly more serious complications and that patients able to cope with DWH lenses were significantly younger than those in the XW group. However, there is an important difference between the proportion of younger patients in our studies. While $77 \%$ of patients in Graham's study were under 70 years, with not a few under 45 years, our DWH patients had a mean age of 70.8 years (58-81) which clearly means that even quite elderly patients can cope with the handling required.

Seventy two per cent of our surviving patients were still wearing their contact lenses after five years and that such a high percentage was achieved may have been due to the fact that patients were in a clinical trial and the amount of encouragement as well as the facilities were exceptional. Our experience showed that more visits were necessary in the first year for patients in either group. Once patients accepted a DWH lens they tended to continue

Table VI Cumulative number of eyes with the commonest recorded complications.

\begin{tabular}{|c|c|c|c|c|c|c|}
\hline \multirow[b]{3}{*}{ Type of complication } & \multicolumn{6}{|c|}{ Time in years } \\
\hline & \multicolumn{2}{|c|}{1} & \multicolumn{2}{|c|}{3} & \multicolumn{2}{|c|}{5} \\
\hline & $D W H$ & $X W$ & $D H W$ & $X W$ & $D W H$ & $X W$ \\
\hline 1. Corneal abscess & 0 & 3 & 0 & 6 & 0 & 8 \\
\hline 2. Infection $\dagger$ & 0 & 3 & 0 & 7 & 1 & 10 \\
\hline 3. Corneal abrasion & 3 & 2 & 4 & 8 & 4 & 8 \\
\hline 4. Punctate epithelial erosion & 0 & 0 & 3 & 4 & 3 & 5 \\
\hline 5. Progressive corneal vascular infiltration & 0 & 1 & 0 & 3 & 0 & 3 \\
\hline $\begin{array}{l}\dagger \text { Relative risk of complications for those wearing } \\
\text { at point }\end{array}$ & & & & & & \\
\hline
\end{tabular}

* Excludes punctate epithelial erosion.

$\dagger=$ Infection in addition to corneal abscess.

$\ddagger=\mathrm{p}<0.01$.

$\S=\mathrm{p}<0.001$.

DWH = Daily-wear hard.

$\mathrm{XW}=$ Extended-wear. 
to do so and 'drop outs' tended to come from the XW group.

The level of after care needed was much greater for $\mathrm{XW}$ patients who required frequent visits to have their lenses cleaned, replaced or to receive attention to complications. The ratio of the number of visits made by XW patients and DWH patient was 3:1.

Eyes in the XW group also made significantly more unscheduled or emergency visits to hospital and that number might have been greater had not the number of scheduled visits been greater because of an awareness of their need to be seen more frequently.

All 'serious' complications increased with time, at least in proportion to the residual numbers still wearing XW lenses. The most disturbing finding was the steady increase in the number of corneal abscesses in the XW group which is the most serious of the complications. After five years the cumulative number of eyes developing an abscess rose to eight which comprised $17 \%$ of the initial cohort. Fortunately, all eyes responded to treatment and no eye in this series was lost. There was a parallel increase in the number of infections of all severity, including symptomatic blepharitis, severe conjunctivitis, and kerato-conjunctivitis suggesting that abscess formation was one end of the spectrum.

Interestingly, some $10 \%$ of eyes were able to make a change from XW to daily-wear at a later stage when the occurrence of complications made it unsafe to continue XW.

There were a variety of reasons to warrant a renewal of an XW lens but the great majority were due to loss or surface contamination. Over the five year period, the mean number of lenses required per eye in the $\mathrm{XW}$ group was two per annum. This was slightly more than had been found by Kersley ${ }^{8}$ and may be due to the fact that different manufactured products were used. In Kersley's study lens cleaning was practised more commonly but in ours lens replacement for contamination was preferred. The mean, however, does not reflectithe fact that many eyes required more than the average number. Table IV, by showing the frequency of lens changes in each treatment group and that XW lenses needed far more in the way of replacement, expresses more fully the big difference between the two groups. While only one eye in the DWH group required eight lenses in five years there were 13 eyes in the XW group which required eight lenses or more in that period and these 13 patients accounted for a total of 146 new lenses. But for the study, such a heavy demand on replacement is neither economical nor is it practical to suggest that these patients should continue using $\mathrm{XW}$ on a long-term basis.

While XW lenses cannot be recommended for long-term aphakic correction because of the complications discussed above, it may however have a place in a scheme of management which allows one to fall back on the use of XW lenses as a temporary measure in cases where DWH lenses cannot be handled or tolerated. In our study, $85 \%$ of patients were fitted with a contact lens, of which half were of the daily wear type even when the average age of patients was over 70 years. Even more encouraging was their ability to continue to manage a DWH lens once tolerated and this capacity should be taken into account in choice of treatment when faced with a difficult or complicated situation at surgery which may prejudice the long-term safety of the eye.

By inference our results would also suggest that in cases where a primary lens implant has not been carried out in cataract surgery, eg after viireous loss, the use of a contact lens is a realistic alternative. The DWH lens should be the lens of first choice and is well tolerated in younger patients. Even if a DWH lens is not tolerated an XW lens can be fitted successfully in the majority, thus buying time for the eye to settle down and for the surgeon to decide on the feasibility or need of a secondary lens implant.

We thank CooperVision and Contact Lenses (MFG) Ltd for supplying the contact lenses and also Allergan Ltd for supplying contact lens solutions.

This study was supported by the National Eye Institute Grant No. EY 02677-09.

\footnotetext{
References

${ }^{1}$ Oxford Cataract Treatment and Evaluation Team: Cataract surgery, interim results and complications of a randomised controlled trial. $\mathrm{Br} \mathrm{J} \mathrm{Oph-}$ thalmol 1986, 70: 402-10.

${ }^{2}$ Bernth-Petersen $\mathrm{P}$ and Sorenson $\mathrm{T}$ : Intraocular lenses versus extended-wear contact lenses in aphakic rehabilitation. Acta Ophthalmol 1983,61: 382-91.
} 
${ }^{3}$ Martin NF, Kracher GP, Stark WJ, Maumenee AE: Extended-wear soft contact lenses for aphakic correction. Arch Ophthalmol 1983, 101: 39-41.

${ }^{4}$ Percival SPB and Yousef KM: Treatment of uniocular aphakia. Br J Ophthalmol 1976, 60: 642-4.

${ }^{5}$ Kersley HJ, Kerr C, Pierse D: Hydrophilic lenses for 'continuous' wear in aphakia: definitive fitting and the problems that occur. Br J Ophthalmol 1977, 61: 38-42.

${ }^{6}$ Stark WJ, Kracher GP, Cowan CL, et al.: Extendedwear contact lenses and intraocular lenses for aphakic correction. Am J Ophthalmol 1979, 88: 535-42.

${ }^{7}$ Graham CM, Dart JKG, Buckley RJ: Extendedwear hydrogel and daily-wear hard contact lenses for aphakia. Ophthalmology 1986, 93: 1489-94.

${ }^{8}$ Graham CM, Dart JKG, Wilson-Holt NW, Buckley $\mathrm{RJ}$ : Prospects for contact lens wear in aphakia. Eye 1988, 2: 48-55.

${ }^{9}$ Kersley HJ and Kerr C. Aphakic extended-wear, one solution to the problems that occur. Contact Intraoc Lens Med J 1981, 7: 57-60. 